Case Reports

\title{
A Unique Presentation of Creutzfeldt-Jakob Disease in a Patient Consuming Deer Antler Velvet
}

\author{
*Joseph Wiedemer, *Yanely Sanchez Ceja, An Cao and Ibrahim Mustafa \\ Department of Neurology, Wright State University Boonshoft School of Medicine, USA
}

\author{
Article history \\ Received: 17-02-2021 \\ Revised: 08-03-2021 \\ Accepted: 13-03-2021 \\ Corresponding Author: \\ Joseph Wiedemer \\ Department of Neurology, \\ Wright State University \\ Boonshoft School of Medicine, \\ USA \\ Email: Wiedemer.2@wright.edu \\ *These authors contributed \\ equally to this work and are \\ considered to be co-first authors
}

\begin{abstract}
Creutzfeldt-Jakob Disease (CJD) is a rare rapidly progressive neurodegenerative prion disorder that is usually sporadic but may also be acquired from exposure to infected sources, classically via infections of bovine or human etiology. Cervid transmission of CJD is of particular concern in North America given the rapid spread of Chronic Wasting Disease (CWD)- the Cervid version of CJD. We present a 61-year-old male patient admitted to our service with a one month history of progressive confusion and gait instability, which led to an initial suspicion of Corticobasal Syndrome (CBS) with unusually rapid progression. CJD was also suspected upon learning that the patient began taking deer antler velvet and bovine colostrum supplements roughly two months prior. The diagnosis of CJD was subsequently confirmed by MRI and RT-QuIC CSF assay. Providers should consider Creutzfeldt-Jakob Disease in the differential diagnosis of a patient with cervid exposure and/or in patients with a presentation resembling corticobasal degeneration, especially if symptom onset is rapid. Although it is unclear how this patient acquired CJD, the potential for prion transmission from cervids to humans should be further investigated by the academic community with considerable urgency.
\end{abstract}

Keywords: Creutzfeldt-Jakob Disease, Prion Disease, Chronic Wasting Disease, Corticobasal Syndrome, Deer Antler Velvet

\section{Introduction}

Creutzfeldt-Jakob Disease (CJD) is a rare, rapidly progressive neurodegenerative prion disorder that is inevitably fatal within a year of diagnosis (Iwasaki, 2017). In the US, 5,212 cases of prion disease demonstrated a median age of death of 67 years, an even gender distribution and an increased annual incidence among those $>65$ years of age between 2003-2015 (Maddox et al., 2020). CJD most-commonly presents in a sporadic form, but is observed in $\sim 15 \%$ of cases as acquired variant-CJD (vCJD), in which prions are transmitted to humans from bovine, cannibalistic, or iatrogenic origins (Iwasaki, 2017; Maddox et al., 2020). Acquired prion disease in humans is also known as Transmissible Spongiform Encephalopathy (TSE) and is most famously exemplified by the bovine-derived TSE outbreak in the United Kingdom-commonly referred to as "mad cow disease"-which occurred in 1986 and peaked in 1993 (Casalone and Hope, 2018).

Prion disease of elk and deer is known as Chronic Wasting Disease (CWD) and is also known to be inevitably fatal (USGS National Wildlife Health Center, 2020; Hannaoui et al., 2017). Unlike human prion disease, CWD is highly contagious among cervids and has been expeditiously spreading throughout North America (USGS National Wildlife Health Center, 2020; Hannaoui et al., 2017; Waddell et al., 2018; Moreno and Telling, 2017). Furthermore, in contrast to CJD, prion disease in cervids typically has a subtle clinical presentation, which makes diagnosis and ecological analyses extremely difficult (Hannaoui et al., 2017; Moreno and Telling, 2017). In the United States, documented cases of CWD have spread from 2 to 26 states since 2000 (USGS National Wildlife Health Center, 2020; Osterholm et al., 2019). Attempts to develop an oral vaccine for livestock against CWD have shown recent promise, but the risk of transmission to humans remains poorly understood (Taschuk et al., 2017). A statewide epidemiological study in Colorado in 2006 was equivocal in discerning an increased risk of CJD in areas heavily impacted by CWD between 1979-2001 (RR 0.81, CI 0.40-1.63) (MaWhinney et al., 2006). Such studies have been unable to determine the risk of prion 
transmission by cervids largely due to the rare frequency of CJD, diagnostic difficulties and variability of symptom onset following exposure (MaWhinney et al., 2006; Nemani et al., 2020).

Although there are no confirmed cases of prion transmission from Cervids to date, there have been several published cases of CJD suspicious for cervid etiology (Angers et al., 2009). The concern of prion transmission from cervids was raised as early as 2001 in a case series that highlighted three separate occurrences of unusually young patients diagnosed with vCJD in the United States (Belay et al., 2001). Two of the patients were frequent hunters and one of the patients was a daughter of a hunter. All three patients had regularly consumed deer and elk meat, which prompted the suspicion for cervid transmission in the absence of any alternative etiology (Belay et al., 2001). Nonetheless, a causal link between the patients' CJD and their histories of cervid exposure could not be definitively concluded (Belay et al., 2001). A comprehensive review by (Waddell et al., 2017) highlighted two additional case series collectively highlighting 5 patients with CJD who had similar exposures to Cervids without any alternative source explaining their disease.

Prions in deer and elk with CWD are primarily derived directly from antler velvet, which is a highly innervated and vascularized tissue layer that sheds cyclically upon antler ossification (Angers et al., 2009). However, CWD-infected cervids are also capable of shedding prions into the environment through feces, urine and saliva (Hannaoui et al., 2017). In all species, prion disease is caused by the conversion of cellular prion protein $\left(\mathrm{PrP}^{\mathrm{C}}\right)$ to a pathological "scrapie" prion protein $\left(\mathrm{PrP}^{\mathrm{SC}}\right)$ which rapidly propagates and accumulates in tissue, primarily in the CNS (Iwasaki, 2017; Moreno and Telling, 2018). In humans, this conversion is believed to be from a sporadic or acquired mutation of the $\operatorname{PrP}^{\mathrm{C}}$ gene PRNP located on the short arm of chromosome 20, which codes for 253 amino acids prior to post-translational modification (Iwasaki, 2017). Multiple mutations of the PRNP gene have been identified that can both cause CJD or increase a host's susceptibility to prion propagation (Iwasaki, 2017; Nemani et al., 2020). Thus, there is growing concern that stronger CWD variants may exist or arise in cervids breaking ostensible species barriers that may exist (Hannaoui et al., 2017; Nemani et al., 2020; Moreno and Telling, 2018).

Several in vivo animal models have demonstrated plausible cervid to human prion transmission, which is most effectively summarized by (Moreno and Telling, 2017; Angers et al., 2009). Most notably, a 2015 study successfully transmitted CWD prions from elk and mule deer via intracerebral injection into transgenic mice expressing human PrP (Kurt and Sigurdson, 2016). Three published studies- the most recent of which in
2014- have demonstrated successful CWD prion transmission to non-human primates (Waddell et al., 2018). The time from prion exposure to symptom onset varied significantly between studies from 70 days to several months (Waddell et al., 2018). Moreover, (Waddell et al., 2018) also highlighted three successful in vitro studies published demonstrating a strong conversion efficiency of CWD prions on human PrP, the most recent of which published in 2015.

\section{Case Report}

A 61 year-old right-handed Caucasian male presented with a one-month history of progressive confusion and gait instability ultimately leading to a 13 day admission. He was found to have moderate cognitive impairment, apathetic affect, left-sided alien hand phenomenon and apraxia, positive Romberg and a dystonic gait pattern with short stride length. The patient was unable to walk without assistance, which prompted an initial suspicion of CBS with unusually rapid progression. The patient also had an extensive history of exotic supplement use going back several years, but notably began taking deer antler velvet and bovine colostrum supplements roughly two months prior. He had no recent travel history, potential occupational exposure to CJD, or family history of prion or neurologic disease. His medical history included hyperlipidemia, distant tobacco use and an incident of diverticulitis several years ago, all of which was considered noncontributory. The patient worked in an office setting, had never been hunting and did not eat deer or elk meet.

Initial Head CT and MRI imaging were unremarkable. A lumbar puncture was performed to evaluate meningitis, autoimmune markers and later 14-3-3 and Rt-QuIC when CJD was suspected (Table 1). A 3-day trial of $1 \mathrm{~g}$ of Methylprednisolone failed to yield symptomatic improvement and the patient's presentation continued to progress. Repeat Brain MRI w/ and w/o contrast on day \#4 of admission demonstrated asymmetric hyperintense cortical ribboning on diffusion-weighted imaging in the right paramedian frontal and parietal cortex as well as a hyperintensity in the right caudate nucleus (Fig. 1). A subsequent EEG demonstrated periodic triphasic waves typical for CJD patients, which was only transiently attenuated by a trial of benzodiazepines without corresponding symptomatic improvement (Fig. 2) (Iwasaki, 2017; Steinhoff et al., 1996). A third MRI obtained on day \#6 of admission showed findings similar to the previous MRI with slightly more hyperintense signal.

The patient continued to demonstrate a progressive decline in neurological status throughout his admission. A Montreal Cognitive Assessment (MoCA) on admission day \#5 yielded a score of 17/30 demonstrating significant cognitive impairment (Fig. 3). After failing to reach a definitive diagnosis despite an extensive workup, 
the patient and his family agreed to discharge to hospice with a likely CJD etiology. Following discharge to hospice, CSF results for protein 14-3-3 and RT-QuIC came back positive, which confirms CJD with $100 \%$ specificity (Iwasaki, 2017). A further post-mortem workup is tentative to date and it remains unclear whether this patient's CJD was sporadic or acquired from exposure to prion-infected cervids or otherwise.

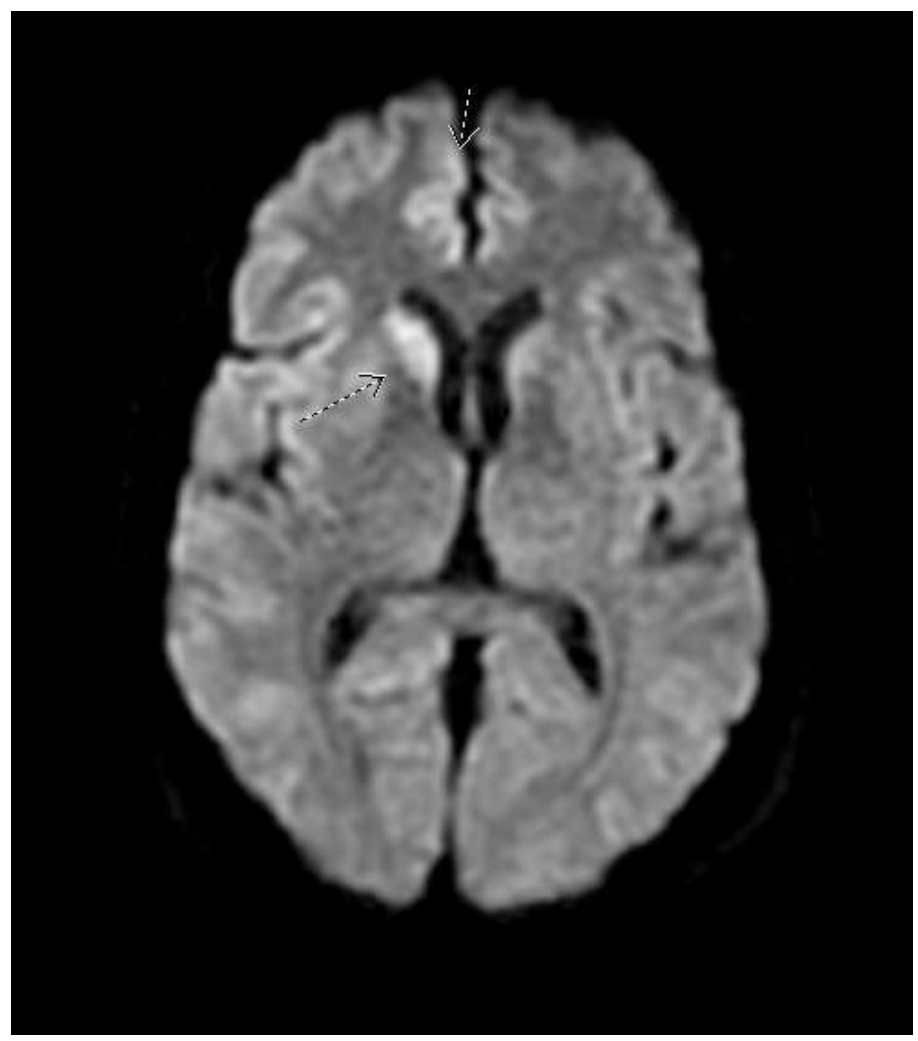

Fig. 1: Axial DWI MRI on day \#4 of admission showing asymmetric hyperintense cortical ribboning on diffusion-weighted imaging in the right paramedian frontal and parietal cortex as well as a hyperintensity in the right caudate nucleus

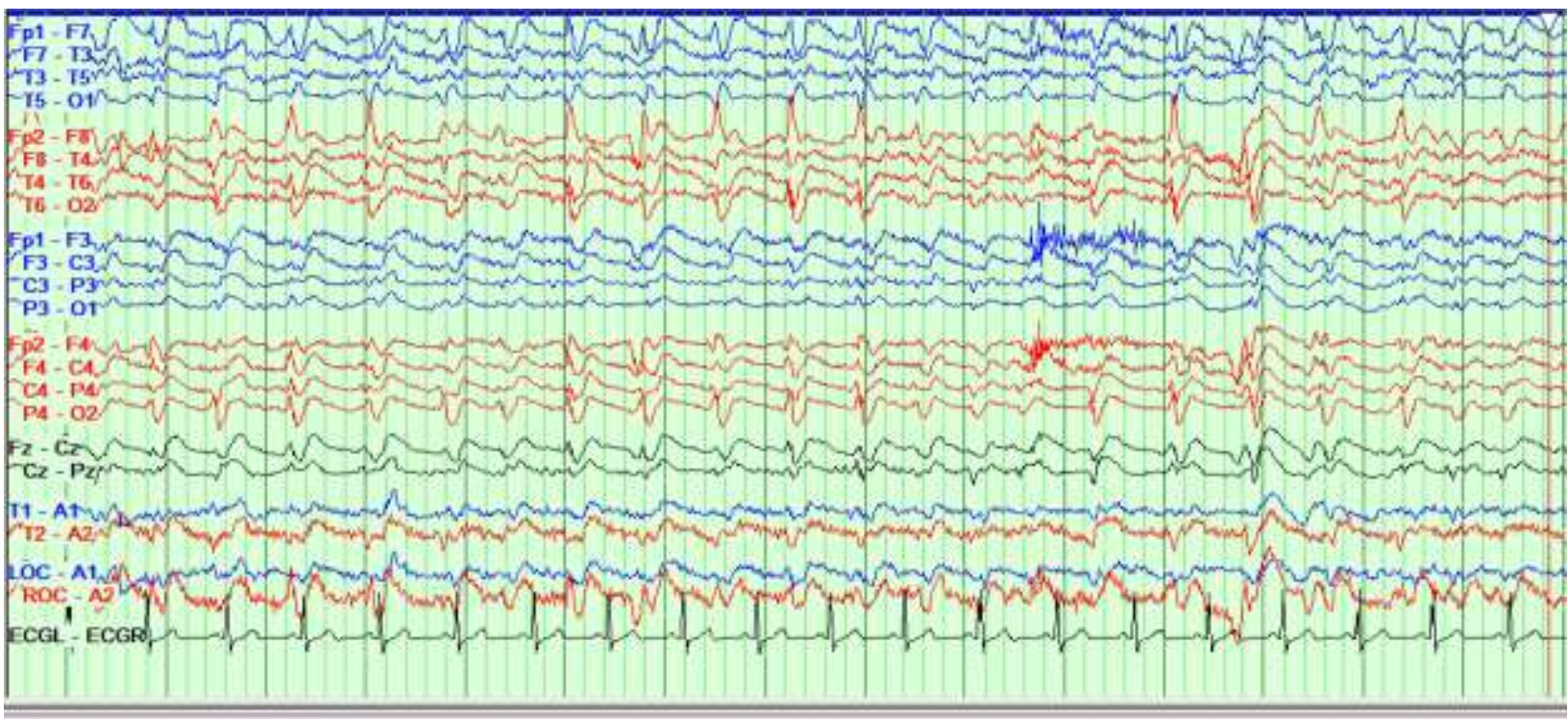

Fig. 2: EEG sample obtained on day \#4 of admission demonstrated diffuse encephalopathic background slowing that was maximal on the right and periodic triphasic waves, typical for CJD patients 


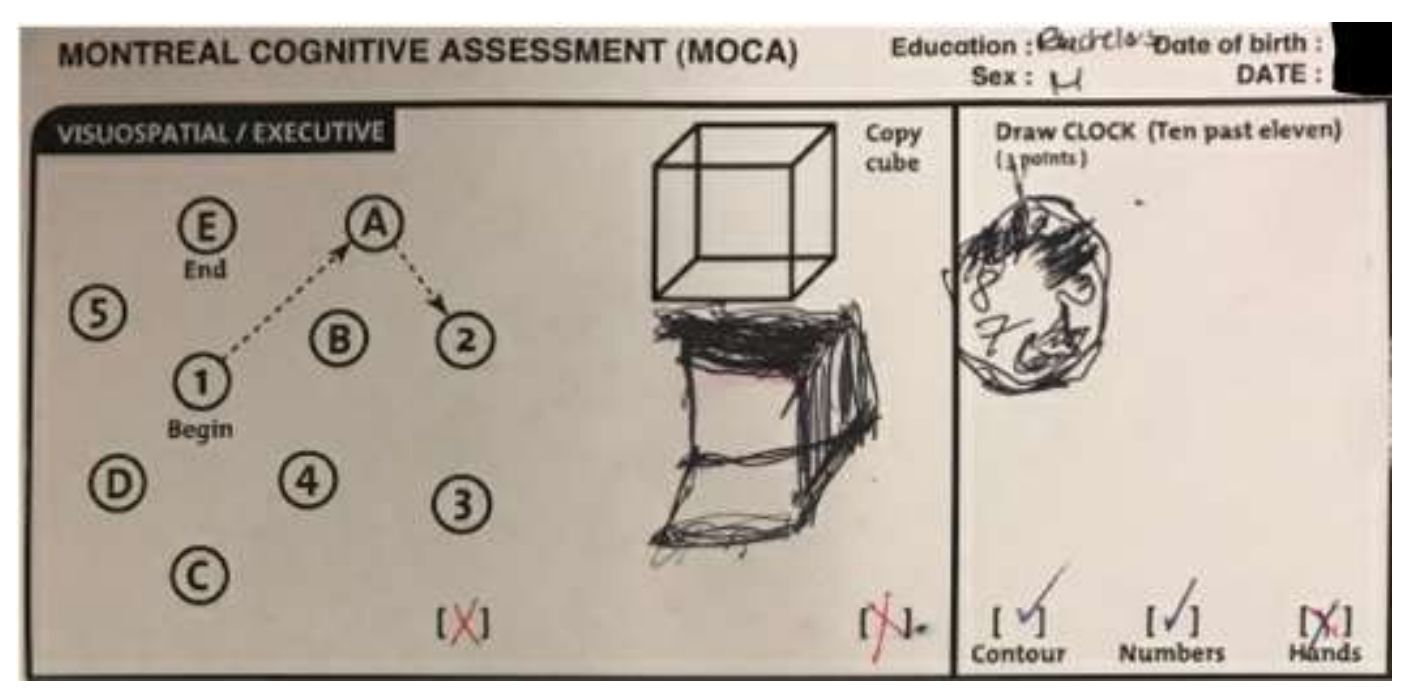

Fig. 3: Excerpt of Montreal Cognitive Assessment (MoCA) performed on admission day \#5 demonstrating significant neurologic regression

Table 1: CSF findings from lumbar puncture performed on admission day \#3

\begin{tabular}{ll}
\hline TUBE NUMBER, CSF & 4 \\
\hline COLOR, CSF & COLORLESS \\
CLARITY, CSF & CLEAR \\
WHITE BLOOD CELLS, CSF 0 - 5 /MM3 & 1 \\
RED BLOOD CELLS, CSF /MM3 & 0 \\
SEGMENTED NEUTROPHILS, CSF \% & 10 \\
BAND NEUTROPHILS \% & 0 \\
LYMPHOCYTES, CSF \% & 60 \\
MONOCYTES, CSF \% & 30 \\
EOSINOPHILS, CSF \% & 0 \\
BASOPHILS, CSF \% & 0 \\
OLIGOCLONAL BANDS COUNTED, CSF & 0 \\
TOTAL CELLS COUNTED, CSF & 10 \\
14-3-3 protein, CSF & POSITIVE \\
RT QuIC, CSF & POSITIVE \\
T-tau protein, CSF & 10416 pg/mL \\
\hline
\end{tabular}

An extensive data query using the lot number and manufacturer information obtained from the patient's supplements revealed no recalls or reported adverse events of any form from either supplement. Investigation into these supplements showed that the deer antler velvet supplement was curated from a farm in Idaho. There are several known areas significantly impacted by CWD near the Idaho border, which is diligently monitored by state and federal agencies (USGS National Wildlife Health Center, 2020).

\section{Discussion}

The bovine-derived CJD outbreak in the United Kingdom of the late 1980s was met with a robust public health and regulatory response and only rare, isolated bovine-derived cases of CJD have been reported in the last 20 years (Iwasaki, 2017). Thus, we believe that it is highly unlikely our patient acquired CJD from the bovine colostrum supplement. Prions specifically from deer antler velvet have been shown to be capable of human transmission through transgenic mouse models, in one case as early as 70 days from inoculation (Moreno and Telling, 2017; Angers et al., 2009). This timeline would support the theory that this patient acquired CJD from the deer antler velvet supplement, as his exposure began at least 60 days prior to the onset of symptoms. It is worth noting that this is the first known case of CJD in which a patient directly consumed deer antler velvet, which is believed to be the primary location of prion propagation in Cervids (Moreno and Telling, 2017; Nemani et al., 2020). This is particularly notable because all other published cases describe cervid exposure from animal meat consumption or hunting.

It is also notable that the patient's clinical presentation was uniquely similar to Corticobasal Degeneration (CBD) with rapid progression for several reasons. Although myoclonus can be appreciated in both diseases, CJD is known to have a significantly more rapid progression and the diseases are largely distinguishable. However, recently published literature has demonstrated that CBD and CJD share many histological similarities (Iwasaki, 2017). Moreover, it is conceivable that a potential case of CJD acquired from Cervids could yield a unique clinical presentation like that observed in bovine TSE (Moreno and Telling, 2017).

\section{Conclusion}

We believe that our patient's case of CJD is highly suspicious for cervid etiology given the circumstances of the case as well as the strong evidence of plausibility reported in published literature. This is the first known 
case of CJD in a patient who had consumed deer antler velvet. Despite the confirmed diagnosis of CJD, a causal relationship between the patient's disease and his consumption of deer antler velvet cannot be definitively concluded.

Supplemental data including molecular tissue sample analysis and autopsy findings could yield further supporting evidence. Given this patient's clinical resemblance to $\mathrm{CBD}$ and the known histological similarities of CBD with CJD, clinicians should consider both diseases in the differential diagnosis of patients with a similarly esoteric presentation. Regardless of the origin of this patient's disease, it is clear that the potential for prion transmission from cervids to humans should be further investigated by the academic community with considerable urgency.

\section{Acknowledgment}

The authors would like to thank their attending physician, Robert Jacoby MD for his exceptional guidance in the care of our patient as well as Emery Steele MD for his outstanding clinical acumen that was instrumental in the diagnosis of our patient.

\section{Author Contributions}

Joseph Wiedemer and Yanely Sanchez Ceja: Drafting/revision of the manuscript for intellectual content, including medical writing for content, Major role in the acquisition of data, Analysis or interpretation of data.

An Cao and Ibrahim Mustafa: Revision of the manuscript for intellectual content, including medical writing for content, Major role in the acquisition of data, Analysis or interpretation of data.

\section{Conflict of Interest}

Each of the authors declare that they have no known competing financial interests, personal relationships, or any other relevant disclosures that could have appeared to influence the work reported in this study.

\section{References}

Angers, R. C., Seward, T. S., Napier, D., Green, M., Hoover, E., Spraker, T., ... \& Telling, G. C. (2009). Chronic wasting disease prions in elk antler velvet. Emerging infectious diseases, 15(5), 696. https://doi.org/10.3201/eid1505.081458

Belay, E. D., Gambetti, P., Schonberger, L. B., Parchi, P., Lyon, D. R., Capellari, S., ... \& Nichols, C. R. (2001). Creutzfeldt-Jakob disease in unusually young patients who consumed venison. Archives of Neurology, 58(10), 1673-1678. https://doi.org/10.1001/archneur.58.10.1673
Casalone, C., \& Hope, J. (2018). Atypical and classic bovine spongiform encephalopathy. In Handbook of clinical neurology (Vol. 153, pp. 121-134). Elsevier. https://doi.org/10.1016/B978-0-44463945-5.00007-6

Hannaoui, S., Schatzl, H. M., \& Gilch, S. (2017). Chronic wasting disease: Emerging prions and their potential risk. PLoS pathogens, 13(11), e1006619. https://doi.org/10.1371/journal.ppat.1006619

Iwasaki, Y. (2017). Creutzfeldt-Jakob disease. Neuropathology, 37(2), 174-188. https://doi.org/10.1111/neup.12355

Kurt, T. D., \& Sigurdson, C. J. (2016). Cross-species transmission of CWD prions. Prion, 10(1), 83-91. https://doi.org/10.1080/19336896.2015.1118603

Maddox, R. A., Person, M. K., Blevins, J. E., Abrams, J. Y., Appleby, B. S., Schonberger, L. B., \& Belay, E. D. (2020). Prion disease incidence in the United States: 2003-2015. Neurology, 94(2), e153-e157. https://doi.org/10.1212/WNL.0000000000008680

MaWhinney, S., Pape, W. J., Forster, J. E., Anderson, C. A., Bosque, P., \& Miller, M. W. (2006). Human prion disease and relative risk associated with chronic wasting disease. Emerging infectious diseases, $12(10), \quad 1527$. https://doi.org/10.3201/eid1210.060019

Moreno, J. A., \& Telling, G. C. (2017). Insights into mechanisms of transmission and pathogenesis from transgenic mouse models of prion diseases. Prions, 219-252. https://doi.org/10.1007/978-14939-7244-9_16

Moreno, J. A., \& Telling, G. C. (2018). Molecular mechanisms of chronic wasting disease prion propagation. Cold Spring Harbor perspectives in medicine, $\quad 8(6), \quad \mathrm{a} 024448$. https://doi.org/10.1101/cshperspect.a024448

Nemani, S. K., Myskiw, J. L., Lamoureux, L., Booth, S. A., \& Sim, V. L. (2020). Exposure Risk of Chronic Wasting Disease in Humans. Viruses, 12(12), 1454. https://doi.org/10.3390/v12121454

Osterholm, M. T., Anderson, C. J., Zabel, M. D., Scheftel, J. M., Moore, K. A., \& Appleby, B. S. (2019). Chronic wasting disease in cervids: implications for prion transmission to humans and other animal species. MBio, 10(4). https://doi.org/10.1128/mBio.01091-19

Steinhoff, B. J., Racker, S., Herrendorf, G., Poser, S., Grosche, S., Zerr, I., ... \& Weber, T. (1996). Accuracy and reliability of periodic sharp wave complexes in Creutzfeldt-Jakob disease. Archives of neurology, 53(2), 162-166. https://doi.org/10.1001/archneur.1996.005500200 74017 
Taschuk, R., Scruten, E., Woodbury, M., Cashman, N., Potter, A., Griebel, P., ... \& Napper, S. (2017). Induction of PrPSc-specific systemic and mucosal immune responses in white-tailed deer with an oral vaccine for chronic wasting disease. Prion, $11(5)$, 368-380. https://doi.org/10.1080/19336896.2017.1367083

USGS National Wildlife Health Center. (2020). Expanding distribution of chronic wasting disease. https://www.usgs.gov/centers/nwhc/science/expandi ng-distribution-chronic-wasting-disease?qtscience_center_objects=0\#qt-science_center_objects
Waddell, L., Greig, J., Mascarenhas, M., Otten, A., Corrin, T., \& Hierlihy, K. (2018). Current evidence on the transmissibility of chronic wasting disease prions to humans - a systematic review. Transboundary and emerging diseases, 65(1), 37-49. https://doi.org/10.1111/tbed.12612 